\title{
Renouvellement de perspective en matière de politique d'informatisation et formalisation d'un nouveau domaine de recherche : le rapport LEMOINE
}

Changing of prospect concerning a policy in Information processing and

definitions of a new sector for research : the Lemoine report.

Dominique Carré

\section{(2) OpenEdition}

\section{Journals}

Édition électronique

URL : http://journals.openedition.org/edc/2789

DOI : 10.4000/edc. 2789

ISSN : 2101-0366

Éditeur

Université Lille-3

Édition imprimée

Date de publication : 1 mai 1991

Pagination : 13-21

ISSN : 1270-6841

\section{Référence électronique}

Dominique Carré, «Renouvellement de perspective en matière de politique d'informatisation et formalisation d'un nouveau domaine de recherche : le rapport LEMOINE », Études de communication [En ligne], 12 | 1991, mis en ligne le 12 janvier 2012, consulté le 03 mai 2019. URL : http://

journals.openedition.org/edc/2789; DOI : 10.4000/edc.2789

Ce document a été généré automatiquement le 3 mai 2019

(c) Tous droits réservés 


\section{Renouvellement de perspective en matière de politique d'informatisation et formalisation d'un nouveau domaine de recherche : le rapport LEMOINE}

Changing of prospect concerning a policy in Information processing and definitions of a new sector for research : the Lemoine report.

\section{Dominique Carré}

1 En 1982 paraît un rapport sur « les technologies d'information - enjeu stratégique pour la modernisation économique et sociale- », connu sous le nom de rapport LEMOINE. Celui-ci revêt deux caractéristiques qui le différencient notoirement du rapport sur « l'informatisation de la société " paru quelques années auparavant, en 1978, plus communément appelé rapport NORA-MINC.

2 Premièrement, le rapport LEMOINE se situe dans la ligne traditionnelle des rapports officiels, à savoir être un moyen de réflexion pour les pouvoirs publics. A contrario, le rapport NORA-MINC qui est aussi un rapport officiel va très vite devenir un outil de travail pour les acteurs institutionnels, économiques et sociaux.

Deuxièmement, le rapport LEMOINE passe quasiment inaperçu du grand public et même de certains responsables. En effet, il ne trouve pas à l'époque de caisse de résonance que cela soit au niveau des médias ou au niveau des acteurs politiques, économiques et sociaux. Le rapport est parfois rejeté par ceux-ci, et au pire, c'est-à-dire dans la majorité des cas, totalement ignoré. A contrario, le rapport NORA-MINC a reçu, quelque temps auparavant, de la presse écrite et audiovisuelle, une fabuleuse couverture médiatique et un accueil intéressé de la part des pouvoirs publics et des acteurs économiques et sociaux. Aujourd'hui, il est devenu une référence obligée pour tous ceux qui s'intéressent au développement des technologies au sein de la société française. L'intérêt porté à ce 
rapport provient sans nul doute du caractère novateur de celui-ci, puisque pour la première fois, en France, un rapport sur une technique prenait également en compte les enjeux et les impacts de cette dernière au niveau de la société globale. On peut dire que le rapport NORA-MINC a servi d'électrochoc en la matière. Pour une fois la technique n'était pas analysée uniquement en des termes strictement technicistes.

Malgré ce constat qui semble jouer en la défaveur du rapport LEMOINE, nous voudrions dans les quelques pages qui vont suivre tenter de montrer et de démontrer que ce rapport est malgré tout important parce qu'il marque un tournant, une ré-orientation de la pensée intellectuelle face au développement de la technique informatique dans la société française, et formalise un nouveau domaine de recherche au sein des sciences sociales.

Pour comprendre la centralité du rapport LEMOINE dans ce renouvellement de perspective, il est nécessaire de re-situer brièvement tout d'abord les tenants et aboutissants du rapport NORA-MINC en matière de politique d'informatisation et de priorités de recherche en sciences sociales, afin de mieux comprendre les réorientations proposées par le rapport LEMOINE, puis d'analyser l'évolution du contexte social et politique entre la parution des deux rapports (1978-1982), ensuite d'examiner les nouvelles orientations préconisées dans le rapport LEMOINE au niveau de la politique informatique française, et enfin de saisir l'impact engendré par celui-ci dans le domaine de la recherche en sciences sociales.

\section{Le rapport Nora-Minc.}

Un peu plus de deux ans après son arrivée au pouvoir Valéry Giscard d'Estaing - Président de la République - confie à Simon Nora - Inspecteur Général des Finances - en date du 20 décembre 1976 le soin de mener à bien une mission d'exploration consistant à « faire progresser la réflexion sur les moyens de conduire l'information de la société ».

7 De ce rapport il ressort :

- Un constat, l'informatique va exploser ; à une technique élitiste va succéder une activité de masse ; c'est la « révolution informatique ».

- Une hypothèse, en se développant l'informatique va se lier aux télécommunications, et ainsi va donner naissance à une nouvelle technique : la télématique.

- Une orientation, le secteur des télécommunications doit devenir le pilier économique indispensable de la conduite de l'informatisation de la société française.

- Des recommandations, pour réussir une telle politique il convient de repenser la stratégie française, en matière d'informatique, à partir d'un nouveau point d'appui qui ne repose plus sur les constructeurs d'ordinateurs, mais sur les spécialistes des télécommunications. En effet pour les auteurs du rapport seul le pôle des télécommunications est en mesure de résister à la stratégie développée par la multinationale IBM dans la gestion des télécommunications. Les pouvoirs publics français doivent pour se faire, constituer un pôle fort autour de l'Administration des télécommunications. Il faut donc renforcer la tutelle étatique dans ce domaine et rassembler des " intervenants divers " autour de cette administration pour concevoir, produire et promouvoir un ensemble de techniques et de produits. La Direction Générale des Télécommunications (D.G.T.) devient ainsi l'élément essentiel de cette stratégie puisqu'elle a pour vocation d'intervenir également sur le plan industriel. C'est à cette époque que la D.G.T. devient un État dans l'État et imprime un véritable volontarisme industriel. 
Tout un dispositif est alors mis en place pour diffuser dans la société française, la prise de conscience du fait informatique : organisation du colloque « informatique et société » en 1979, lancement de la collection intitulée " informatisation et société " à la Documentation Française ${ }^{1}$, entre autres. Des mesures sont également prises pour faciliter l'instauration de cette politique : conception et lancement d'un plan télématique, en 1978 ; naissance de l'Agence de l'Informatique (A.D.I.) qui a pour vocation la création, la diffusion et l'utilisation des techniques informatiques en vue de moderniser le tissu économique et social ; annonce par le Ministre de l'Éducation nationale, Christian Beullac, en 1979, de l'opération des 10000 micro-ordinateurs dans les écoles; de nombreuses études sont financées au niveau sectoriel sur l'informatisation ${ }^{2}$, ou au niveau transversal sur la télématique ; enfin des expérimentations sont lancées: la première en 1980 à SaintMalo (Ile et Vilaine) pour tester la version vidéotex de l'annuaire électronique, la deuxième sur le site de Vélizy (banlieue parisienne) où 2500 ménages expérimenteront, progressivement, à partir de juin 1981, une application du vidéotex interactif à travers le système Télétel.

9 Quant aux orientations de la recherche, elles sont limpides même si elles ne sont pas clairement définies puisque le rapport NORA-MINC fixe un cadre idéologique précis et incontournable celui des " enjeux culturels ", et sollicite informellement les chercheurs en sciences sociales à mener des investigations, des études sur les répercussions, impacts engendrés par la technique sur le social, le culturel et l'économique.

\section{L'évolution du contexte social et politique (1978-1982).}

10 Le septennat de Valéry Giscard d'Estaing repose sur un système politique que l'on peut baptiser de "libéral avancé » et se caractérise par trois éléments clefs en matière d'informatique, de télécommunication et d'audiovisuel :

- La volonté de déployer une politique libérale qui privilégie le marché et la libre concurrence. C'est à cette époque que la loi de réforme de l'audiovisuel est adoptée. L'objectif est alors d'éclater l'ORTF en sept sociétés et d'introduire une concurrence au sein du service public.

- La nécessité de mener une politique interventionniste des pouvoirs publics pour rattraper un retard social et économique. C'est le cas du téléphone. En 1974, 20 \% des familles françaises possèdent un poste téléphonique, alors qu'en 198170 \% sont équipés. Mais aussi pour développer des secteurs d'activités face à un contexte international peu propice. Le renforcement du rôle de l'Administration des télécommunications en est un excellent exemple.

- La recherche de moyens pour garantir la vie privée des citoyens contre les débordements ou des utilisations perverses de certaines applications informatiques. Dans ce domaine, la loi du 6 janvier 1978 relative aux fichiers et aux libertés est adoptée et une Commission Nationale de l'Informatique et des Libertés est instaurée (CNIL).

L'arrivée d'un gouvernement de gauche "social démocrate ", en 1981, ne va guère changer la politique française en matière d'informatique et de télécommunications. Certes, on assiste à la mise en place d'une planification industrielle, au renforcement de la tutelle étatique et à une redistribution des cartes dans le secteur de l'informatique autour du groupe BULL ; mais la politique informatique et télématique est toujours pilotée par 
l'amont. C'est-à-dire, que la politique industrielle prime, même si elle s'organise et s'accompagne d'une dimension sociale, afin de lutter contre les dégâts du progrès (CFDT, 1977) ou pour favoriser l'intervention des salariés et de leurs représentants sur les changements technologiques au sein des entreprises - lois Auroux ${ }^{3}$.

La politique du gouvernement se structure, alors, autour de la «filière électronique ». Le concept de «filière électronique » a été développé, fin des années 1970, principalement par O. Pastré et J.H. Lorenzi. Rapidement dit le concept de « filière » est directement lié à une hypothèse néo-fordienne concernant l'issue de la crise économique. Ainsi la « filière électronique » en modifiant à la fois la manière de produire et la manière de consommer, aurait cette vertu particulière de transformer la norme de production (innovation de procédés) et la norme de consommation (innovation de produits et de services), créant ainsi des conditions favorables à une restauration du régime d'accumulation inspiré par le fordisme qui était entré en crise au milieu des années 1970. La relance néo-fordienne ayant besoin d'un nouveau consensus social sur le progrès technique. Elle requiert, donc, une forme de concertation sociale appropriée. La " filière électronique » va devenir le mot clef de la politique industrielle du premier gouvernement socialiste français, après 1981.

13 Au niveau de la recherche, les pouvoirs publics vont concevoir des programmes "mobilisateurs " pour orienter et développer certains axes de recherche. A titre d'exemple, le travail mené par le Programme Mobilisateur « Technologie, Emploi, Travail » est révélateur de ce type de politique.

\section{Renouvellement de perspective en matière de conduite de la politique industrielle informatique.}

Par une lettre du 7 septembre 1982 Pierre Mauroy - Premier Ministre - confie à Philippe Lemoine - Commissaire Général auprès de la CNIL et secrétaire du Programme mobilisateur « Technologie, Emploi, Travail » - une mission d'étude pour définir un cadre administratif pour assurer la mise en oeuvre d'une politique d'utilisation des techniques informatiques.

Conformémment au contexte idéologique ambiant, le rapport LEMOINE ${ }^{4} s^{\prime}$ inscrit dans une approche où progrès technique et progrès social sont liés ; mais l'auteur de ce rapport ne propose pas seulement d'articuler et de conjuguer politique industrielle et politique d'utilisation. Il va bien au-delà et dresse un cadre conceptuel d'ensemble pour mettre en oeuvre une politique reposant sur le versant « utilisation » des technologies, tout en favorisant la démocratisation des choix technologiques. Sur le plan industriel, il préconise une «nouvelle » politique industrielle qui ne serait plus pilotée par l'amont, mais par l'aval. C'est-à-dire, répondant avant tout aux besoins des utilisateurs de l'informatique et de la télématique.

Le rapport LEMOINE renverse les polarisations existantes. Tout d'abord la priorité en matière de politique industrielle informatique ne doit plus être donnée aux " matériels " mais aux « logiciels et progiciels ». Ensuite une logique de services et de contenus doit supplanter une logique d'équipements techniques ou de produits conçus en laboratoire. Enfin la gestion sociale de la technique ne doit plus s'effectuer en aval, mais en amont, afin d'associer les futurs utilisateurs à la conception de leurs propres systèmes d'information. 
17 En d'autres termes le pôle utilisation sociale et culturelle doit devenir le moteur du pôle industriel, cela signifie également que le système d'action mis en place, préalablement, par les pouvoirs publics est doublement dépassé : premièrement, la notion de " filière électronique " est à revoir, et deuxièmement le rôle assigné aux diverses organisations administratives n'est plus pertinent. Certes l'État français doit toujours être le pilier d'une politique volontariste, mais cette dernière doit être repensée. L'État ne peut plus " interdire ou réglementer ", ni "faire et expérimenter », encore moins « faire faire », son nouveau rôle doit consister à prendre des mesures pour « savoir faire » et « faire savoir ». Cette voie, semble la seule satisfaisante pour déployer une véritable politique fondée sur $1^{\prime}$ '« utilisation » des techniques. Pour mener à bien une telle politique, les pouvoirs publics doivent assurer une fonction d' « écoute » et de « médiation » entre les réalités du terrain et les plus hautes instances de décision stratégique.

ur le plan social, le rapport LEMOINE propose de donner la parole aux utilisateurs, de mettre en oeuvre des actions intensives de formation, de favoriser les débats sur la place publique.

Contrairement au rapport NORA-MINC, les recommandations du rapport LEMOINE ne seront guère reprises par les pouvoirs publics. Malgré cela on peut affirmer que le rapport LEMOINE a eu un mérite essentiel, celui d'esquisser un nouveau cadre théorique de la politique française en matière d'informatisation ; mais il a eu un tort considérable, à savoir de proposer une approche moins habituelle et prestigieuse de la technologie qui n'était pas partagée par le gouvernement de l'époque et qui heurtait encore plus les schémas traditionnels de raisonnements technicistes de la techno-structure des grands corps (polytechnique, mines etc... ). Notre analyse semble être en grande partie confirmée par les propos tenus par P. Lemoine et X. Dalloz en 1990, dans la revue Médiaspouvoirs ${ }^{5}$, sur la politique française passée et actuelle en matière de technologie de l'information et de la communication. Ces deux auteurs critiquent sévèrement la politique actuelle suivie par la France en matière de TV à haute définition. Une fois de plus disent-ils «l'approche française prônée, repose strictement sur la technique, et un volontarisme colbertiste qui est totalement inadapté au contexte économique et social ».

\section{Impact du rapport Lemoine sur la recherche en sciences sociales.}

Le rapport LEMOINE a certainement suscité un intérêt plus grand de la part des chercheurs en sciences sociales, notamment à travers l'intérêt que portait ce rapport au concept d'expérimentation sociale de la technique. En effet pour P. Lemoine l'un des moyens privilégiés pour mieux connaître les besoins des futurs utilisateurs consiste à recourir à l'expérimentation sociale d'une technique afin que la demande et les usages supplantent l'offre.

21 Le rapport LEMOINE n'a pas donné un cadre précis de recherche, mais plutôt une nébuleuse d'interrogations qui a progressivement formalisé de nouvelles problématiques de recherche. Il est à noter que ces esquisses de problématiques avaient déjà pu voir le jour en amont du rapport LEMOINE dans le rapport MATTELART-STOURDZÉ sur la « Technologie, culture et communication » et dans les études réalisées par des sociologues en 1982-83' dans le cadre des expériences Teletel 3V et du CESIA. A titre d'exemples : Teletel et l'Administration - étude sur les comportements d'un échantillon d'usagers et 
sur la logique de production des services vidéotex, la télématique en milieu rural expérience de télé-informations dans les Alpes de Haute-Provence et dans le Lot et Garonne -, entre autres.

Nous ne prétendons pas, que de ce rapport soient sortis de nouveaux axes de recherche, tout au plus nous faisons l'hypothèse qu'il a permis de formaliser un renversement de problématique, et par là même, a servi à dynamiser, ou catalyser la recherche en sciences sociales sur les politiques d'utilisation, la formation des usages et les modalités de diffusion des technologies. Au point que les études sur les usages ont représenté le courant dominant de la recherche en sciences sociales sur le vidéotex ${ }^{7}$ et que la Mission TV -Câble finança de nombreuses études sur les politiques d'utilisation du câble et le développement des usages.

\section{Conclusion}

De notre analyse, il ressort que le rapport NORA-MINC a eu principalement un rôle de " révélateur ", de " prise de conscience " du fait informatique, et une portée médiatique considérable. Alors que le rapport LEMOINE contribua à un renouvellement de perspective dans le domaine de la pensée politique industrielle et économique française en matière d'informatique, mais aussi dans le domaine de la recherche en sciences sociales.

Sur le plan économique : le rapport LEMOINE proposa un renversement de la problématique générale en matière de politique de développement de l'informatique dans la société. Cette nouvelle politique est fondée sur une approche "utilisation " de la technique informatique au détriment de la politique «industrielle» traditionnellement menée jusqu'alors.

Sur le plan recherche en sciences sociales : il formalisa un nouveau domaine de recherche, celui des usages sociaux.

\section{BIBLIOGRAPHIE}

CFDT, (1977), Les dégâts du progrès, Paris, Seuil.

Lemoine, P., (1983), Les technologies d'information enjeu stratégique pour la modernisation économique et sociale, Paris, La Documentation Française.

Lorenzi, J.M., Pastre, O. \& Toledano, J., (1980), La crise du vingtième siècle, Paris, Économica.

Mattelart, A. \& Stourdzé, Y., (1982), Technologie, culture et communication, Paris, La Documentation Française.

Nora, S. \& Minc, A., (1978), L'informatisation de la société, Paris, Seuil. 


\section{NOTES}

1. A titre d'exemples, voici quelques titres publiés dans cette collection : Les chiffres clefs de l'informatisation. L'information demain - de la presse écrite aux nouveaux médias -. Les enjeux culturels de l'informatisation. Négocier l'ordinateur? La concertation sur les nouvelles technologies dans l'entreprise.

2. Dans l'Éducation nationale, par exemple : Rapport SIMON «L'éducation et l'informatisation de la société » (1980), rapport TEBEKA “Formation des spécialistes informaticiens » (1981), rapport SCHWARTZ «L'informatique et l'éducation » (1981).

3. Les lois « Auroux » datent de 1982, deux articles nous intéressent plus particulièrement : art. L 432-2 du code du travail, le comité d'entreprise est informé et consulté, préalablement à tout projet important d'introduction de nouvelles technologies... ; art. L 434-6 le comité d'entreprise peut avoir recours à un expert en nouvelles technologies...

4. Il est intéressant de constater que sur la page de garde, un sous-titre est présent : " Propositions pour une politique d'utilisation ». Alors que ce dernier n'apparaît pas sur la première de couverture.

5. LEMOINE, P., DALlOZ, X (1990), « Pour des politiques enfin" Haute définition", Médiaspouvoirs, $\mathrm{n}^{\circ}$ 20, octobre-décembre, pp. 153-166. Dans cet article une phrase confirme notre propos : « L'un des auteurs a quitté l'Administration en 1985 (il s'agit de P. Lemoine) après un rapport au Premier Ministre où il recommandait d'abandonner pour l'informatique le modèle de l'État protectionniste et acheteur au profit d'une stratégie technologique liée à la modernisation économique et sociale ".

6. Cf. les travaux de Ponjaert, Georgiades, Magnier, Marchand, Ancelin, Jouët, Eksl, Gauthronet, entre autres.

7. Comité de rédaction des revues TIS et RÉSEAUX (1989), « Éditorial », revue TIS, volume 2, n 1 , et revue RÉSEAUX, n³7, pp. 9-13.

\section{RÉSUMÉS}

Succédant au rapport Nora Minc (1978) qui avait appelé à une révolution informatique et avait mobilisé industriels et gouvernement pour le développement d'une "filière électronique " autour des Télécommunications, le rapport de Ph.Lemoine, "Les technologies d'information, enjeu stratégique pour la modernisation économique et sociale » (1982) est passé, lui, inaperçu.

Mettant l'accent sur les usages, les services, les logiciels, il appelait à un renversement de perspective, en prônant un pilotage de la politique industrielle "par l'aval ». Si le rapport Lemoine n'a pas reçu d'écho favorable, il a néanmoins formalisé un nouveau domaine de recherche en sciences sociales : celui des « usages sociaux », qui s'est notablement développé lors des études sur le vidéotex. Pour cela il fait date.

Following the Nora Minc report (1978) which had called for a data processing revolution and had required the attention of the industrials and the government about the development of a " processing connexion » with the help of telecommunications, the report from Ph.Lemoine " The technologies of information as a strategie wager for economical and social modernization » was left unnoticed. 
Putting the stress on the uses, services and software this report was calling for a reversal of prospects, by recommending the consumers demand as superior to the industrial offer. Though the Lemoine report did not draw a proper attention, it gave the definition of a new sector in social sciences : that of social usefulness which progressed noticeably, along with the studies about videotex. That is the reason why it is considered as important.

INDEX

Mots-clés : politique publique, NTIC, informatisation, politique industrielle, étude d'usages, télécommunications

Keywords : public policy, NICT, computerization, industrial policy, usage studies, telecommunications

\section{AUTEUR}

\section{DOMINIQUE CARRE}

Dominique Carré, Enseignant à l'Université Paris-Nord, Chercheur au CENT 